\title{
C/EBP homologous protein induces mesangial cell apoptosis under hyperglycemia
}

\author{
DAQIANG HE, JIANQIONG LI, JIAYAO ZHAO, JING FEI and XIAOMING ZHANG
}

\begin{abstract}
Department of Anatomy and Cell Biology, School of Medicine, Zhejiang University, Zhejiang 310058, P.R. China
\end{abstract}
Received August 6, 2012; Accepted December 7, 2012

DOI: $10.3892 / \mathrm{mmr} .2012 .1234$

\begin{abstract}
Diabetes mellitus is known to cause kidney impairment; however, the mechanism remains elusive. The aim of this study was to investigate the role of $\mathrm{C} / \mathrm{EBP}$ homologous protein (CHOP), an important protein in endoplasmic reticulum stress-mediated mesangial cell apoptosis in hyperglycemia. Mesangial cells were cultured in normal (control group) and high glucose medium (high glucose group). TUNEL staining was performed to assess apoptotic cells in the groups. The expression of CHOP and caspase-3 was also assayed by immunohistochemistry and western blot analysis. Following $24 \mathrm{~h}$ culture in high glucose medium, TUNEL-positive cells were observed to be significantly increased $(\mathrm{P}<0.01)$. The expression of CHOP and caspase-3 in mesangial cells was also found to be significantly enhanced under high glucose conditions compared with the normal group $(\mathrm{P}<0.01)$. The results indicate that CHOP mediates apoptosis in mesangial cells under hyperglycemia and may play a role in the development of diabetic nephropathy.
\end{abstract}

\section{Introduction}

Diabetic nephropathy is the leading cause of end stage renal disease (1-3). A number of studies have reported that hyperglycemia activates multiple downstream signaling pathways in the diabetic kidney leading to extracellular matrix accumulation, endothelium dysfunction, glomerular hyperfiltration and eventually induction of glomerular hypertrophy, increased glomerular basement membrane thickness and interstitial fibrosis (4-5). Mesangial cell response to pathological stimuli is associated with the main events of glomerular injury and is important for resistance to glycoxidative stress through an antioxidant response (6). A previous study reported that alterations

Correspondence to: Dr Xiaoming Zhang, Department of Anatomy and Cell Biology, School of Medicine, Zhejiang University, 866 Yuhangtang Road, Hangzhou 310058, P.R. China

E-mail: zxm@zju.edu.cn

Key words: C/EBP homology protein, caspase-3, apoptosis, hyperglycemia, mesangial cells in glucose associated with diabetes mellitus induce mesangial cell apoptosis and contribute to diabetic nephropathy (7). However, the mechanism by which mesangial cell apoptosis is regulated in diabetic individuals remains unclear.

The endoplasmic reticulum (ER) is recognized as an organelle involved in the folding of secretory and membrane proteins. An additional emerging function of the ER is the regulation of apoptosis in the cell (8). A number of stimuli, including ischemia, hypoxia, hyperglycemia, gene mutation and elevated protein synthesis, are collectively referred to as ER stress and all potentially cause ER dysfunction $(9,10)$. ER stress has been reported to be critical to the pathogenesis of a number of acute and chronic kidney diseases, including renal ischemia, acute kidney injury and diabetic kidney disease (11). Stimuli that increase the demand on the ER to synthesize proteins or degrade improperly folded proteins cause this stress. Several components of the diabetic milieu, i.e., high glucose, free fatty acids, albumin, oxidative activity and inflammation, induce ER stress in numerous tissues (12-14). However, prolonged activation of ER stress ultimately initiates the apoptotic pathway. $\mathrm{C} / \mathrm{EBP}$ homologous protein $(\mathrm{CHOP})$ is an important protein in ER stress-mediated apoptosis (15). A number of previous studies have demonstrated that the ER is the major cell death organelle in cadmium-induced mesangial cell apoptosis (16), glomerular and tubular damage in kidney disease and renal injury $(17,18)$.

Therefore, we hypothesized that mesangial cells suffer from high glucose, induction of CHOP-mediated apoptosis in the kidney and eventually develop diabetic nephropathy. In the present study, mesangial cells were cultured in a high glucose medium to determine the association of CHOP-mediated cell apoptosis in vitro.

\section{Materials and methods}

Cell culture. Mesangial cells of the renal gloraerule (HBZY; cx0310, Wuhan Boster Bio-Engineering Limited Company, Wuhan, China) were seeded into 24-well cell culture plates in DMEM/F12 with $10 \% \mathrm{FBS}$ and $0.05 \mathrm{mM}$ glutamine to observe survival and neurite outgrowth. All culture reagents were purchased from Gibco-BRL (Grand Island, NY, USA). Mesangial cells were cultured to day 6 and then the medium was replaced with a high glucose DMEM/F12 medium for $24 \mathrm{~h}$. The high glucose group received $100 \mathrm{mM}$ glucose and the control group received basal $25 \mathrm{mM}$ glucose. The study 
A

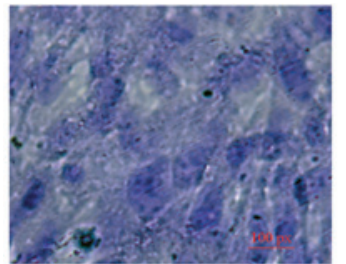

B

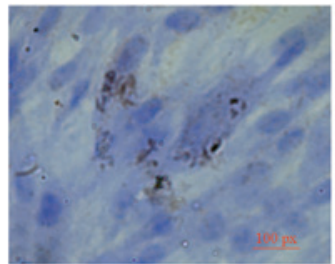

Figure 1. TUNEL staining in mesangial cells with (A) normal and (B) high glucose medium (magnification, $\mathrm{x} 400$ ).
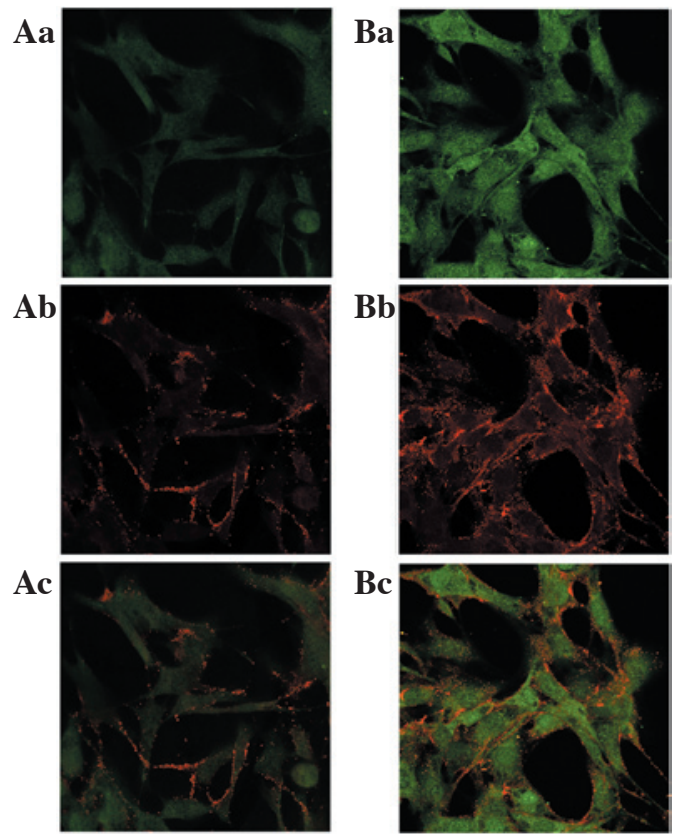

Figure 2. Expression of (a) CHOP and (b) caspase-3 in mesangial cells with (A) normal and (B) high glucose medium. (c) Merged images (magnification, x600). CHOP, C/EBP homologous protein.

was approved by the ethics committee of School of Medicine, Zhejiang University, Hangzhou, China.

TUNEL staining analysis. TUNEL staining was performed to assess apoptotic cells in cultured mesangial cells according to the manufacturer's instructions. Cells labeled with brown were counted as positive cells under a microscope.

Immunohistochemistry and immunofluorescence of $\mathrm{CHOP}$ and caspase-3 protein in cultured mesangial cells. For immunohistochemical analysis, slides of cultured mesangial cells of the two groups were washed in $0.01 \mathrm{M}$ phosphate buffered saline (PBS) containing 0.3\% Triton X-100 (pH 7.4, PBS-T), then immersed in $2 \%$ normal goat serum in PBS for $2 \mathrm{~h}$ at $37^{\circ} \mathrm{C}$. Slides were incubated overnight at $4^{\circ} \mathrm{C}$ with polyclonal CHOP and caspase-3 antibodies (both 1:100; Santa Cruz Biotechnology, Santa Cruz, CA, USA) in PBS containing 1\% bovine serum albumin. Following this, slides were washed in PBS (3x5 min), incubated with biotinylated goat-anti-rabbit IgG (1:200; Wuhan Boster Bio-Engineering Limited Company) in PBS for $2 \mathrm{~h}$ at room temperature, washed in PBS-T (3x5 min) and incubated in avidin-biotin-peroxidase complex solution (1:100; Wuhan Boster Bio-Engineering Limited Company) for $2 \mathrm{~h}$ at room temperature. Slides were
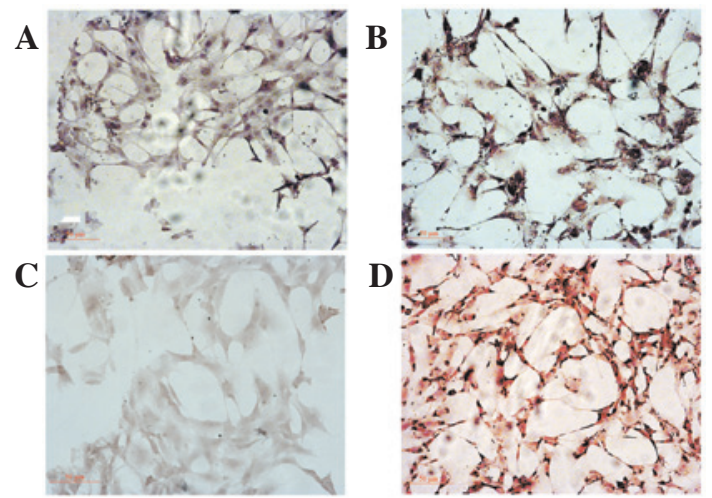

Figure 3. Immunohistochemical analysis of CHOP and caspase-3 in mesangial cells with (A and C) control and (B and D) high glucose medium. CHOP, $\mathrm{C} / \mathrm{EBP}$ homologous protein.

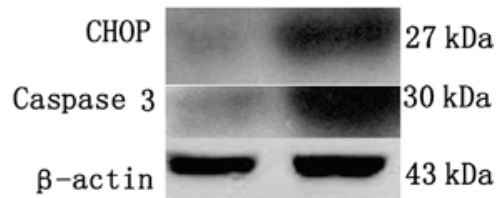

Figure 4. Western blot analysis for $\mathrm{CHOP}$ and caspase-3 in mesangial cells in normal and high glucose medium. CHOP, C/EBP homologous protein.

then rinsed again in PBS-T (3x5 min). Immunolabelling was visualized with $0.05 \%$ diaminobenzdine and $0.3 \% \mathrm{H}_{2} \mathrm{O}_{2}$ in PBS. Following staining, the sections were counterstained with hematoxylin and then dehydrated with ethanol and xylene prior to application of a coverslip with Permount. Rat immunoglobulin IgG (1:200; Biomeda Corporation, Foster City, CA, USA) was used instead of primary antibody as a negative control. Immunofluorescent microscopy was performed using the Zeiss LSM-510 confocal microscope (63X/1.2 W; Carl Zeiss Microscopy, LLC, Thornwood, NY, USA).

Western blot analysis of CHOP and caspase-3 protein in cultured mesangial cells. Total protein samples were obtained from cultured cells and the concentration of protein was determined by the Lowry method using a Bio-Rad DC protein assay kit (Hercules, CA, USA). Cell lysates containing equal amounts of protein $(50 \mu \mathrm{g})$ were resolved by $8-10 \%$ SDS-polyacrylamide gel electrophoresis and then transferred onto Millipore nitrocellulose membranes (Billerica, MA, USA). Next, the blots were blocked with a solution containing 5\% skimmed milk in Tris-buffered saline with $0.05 \%$ Tween-20 (TBST) for $1 \mathrm{~h}$ at room temperature and treated with primary antibodies (polyclonal CHOP and caspase-3 antibodies; 1:2,000; Santa Cruz Biotechnology) in TBST overnight at $4^{\circ} \mathrm{C}$, washed for $1 \mathrm{~h}$ with TBST and further probed with secondary HRP-conjugated anti-rabbit, anti-mouse or anti-goat IgG $(1: 2,000)$ in TBST for $1 \mathrm{~h}$ at room temperature. Immune complexes were visualized using an ECL detection system according to the manufacturer's instructions.

Statistical analysis. Sections were observed and analyzed at x400 magnification with UTHSCSA Image Tools 3.0 
(University of Texas Medical School, San Antonio, TX, USA) and the number and optical density of the CHOP and caspase-3-positive cells were determined. A probability of $95 \%$ was considered to indicate a statistically significant difference. Data are presented as mean \pm SD.

\section{Results}

TUNEL staining assay in two groups. TUNEL-positive cells revealed brown staining in the nucleus. The number of TUNEL-positive cells increased in mesangial cells in high glucose medium compared with normal (25.2 \pm 7.7 vs. $3.3 \pm 1.6 \%$; $\mathrm{P}<0.05$; Fig. 1). Results indicate that mesangial cells are selectively susceptible to apoptosis under hyperglycemic conditions.

Immunofluorescence. The CHOP positive fluorescence signal was localized to the nucleus and an upregulation of $\mathrm{CHOP}$ staining was observed in the mesangial cells cultured in high glucose medium while cells cultured in normal medium were negative for CHOP expression. Expression of caspase-3 was also increased in high glucose treated cells (Fig. 2; $\mathrm{P}<0.05$ ).

Immunochemistry analysis of $\mathrm{CHOP}$ and caspase-3 expression. Immunochemical analysis revealed that $\mathrm{CHOP}$ and caspase-3 were expressed at high levels in mesangial cells cultured in high glucose medium while cells in the normal group exhibited modest or weak immunoreactivity for $\mathrm{CHOP}$ and caspase-3 protein (Fig. 3). The percentage of CHOP- and caspase-3-positive cells in mesangial cells in high glucose medium was found to be significantly higher $(42.9 \pm 14.2$ and $58.1 \pm 17.6 \%)$ than that of the normal group $(13.7 \pm 3.5$ and $6.8 \pm 1.1 \% ; \mathrm{P}<0.05)$

Western blot analysis of CHOP and caspase-3. Western blot analysis revealed single bands of $27 \mathrm{kDa}$. Densitometric analysis of bands corresponding to CHOP and caspase-3 demonstrated a significant increase in relative protein content in mesangial cells in high glucose medium $(375.3 \pm 78.4$ and $350.2 \pm 64.2 \%$ ) compared with the normal group (Fig. 4; $\mathrm{P}<0.05)$.

\section{Discussion}

CHOP was previously identified as an ER stress-induced transcription factor that functions as a significant mediator of apoptosis in response to ER stress (19). CHOP gene induction is primarily mediated through the PERK/eIF2 $\alpha / A T F 4$ UPR pathway, although IRE1 $\alpha / \mathrm{XBP} 1$ and ATF6 $\alpha$ pathways also contribute $(20,21)$. CHOP is a well-known inhibitor of gene transcription and functions primarily through dimerization with and negative regulation of other leucine zipper transcription factors of the C/EBP and CREB families. Overexpression of CHOP leads to growth arrest and apoptosis (22). Thus, induction of CHOP indicates that ER-initiated apoptosis is induced (23) and our previous study revealed that CHOP plays a role in renal injury (18). Caspase-3 is a downstream target of CHOP and the UPR-independent pathways were demonstrated to culminate in executioner caspase-3 activation and apoptosis in a previous study of proteasome inhibitor-mediated apoptosis (24). Caspase-3 is the final caspase of the apoptotic cascade which activates cytolytic enzymes responsible for apoptosis (25).

The mesangium occupies a central anatomical position in the glomerulus, located between the fenestrated endothelial lining of the capillary lumen and the glomerular basement membrane. This structure is involved in the pathology of all types of chronic, progressive kidney diseases (26). The mesangium is composed of mesangial cells embedded in the mesangial matrix, providing structural support to capillary loops and modulating glomerular filtration (27). Mesangial cell responses to pathological stimuli are associated with the main events of glomerular injury, leukocyte infiltration, cell proliferation and NF- $\kappa \mathrm{B}$ and MCP-1 expression $(26,28)$. The present study demonstrated that the number of TUNEL-positive mesangial cells was significantly increased under high glucose conditions and CHOP was simultaneously induced. The induction of CHOP in mesangial cells promoted ER-initiated apoptosis under these conditions, induced interstitial fibrosis and enhanced progression of diabetic nephropathy.

In conclusion, the current study demonstrates that ER stress mediates apoptosis in mesangial cells under hyperglycemia. The results indicate that ER-initiated apoptosis may contribute to mesangial cell apoptosis and play a role in the development of diabetic nephropathy. However, further studies should be performed to determine the intracellular signaling pathways involved in ER stress-induced fibrosis in diabetic nephropathy.

\section{Acknowledgements}

The present study was supported by the National Natural Science Foundation of China (no.30971124).

\section{References}

1. Tokodai K, Amada N, Kikuchi H, Haga I, Takayama T and Nakamura A: Outcomes of renal transplantation after end-stage renal disease due to diabetic nephropathy: a single-center experience. Transplant Proc 44: 77-79, 2012.

2. van den Berg E, Hospers FA, Navis G, et al: Dietary acid load and rapid progression to end-stage renal disease of diabetic nephropathy in Westernized South Asian people. J Nephrol 24: 11-17, 2011

3. Martínez-Castelao A, De Alvaro F and Górriz JL: Epidemiology of diabetic nephropathy in Spain. Kidney Int Suppl: S20-S24, 2005.

4. Samnegard B, Jacobson SH, Jaremko G, et al: C-peptide prevents glomerular hypertrophy and mesangial matrix expansion in diabetic rats. Nephrol Dial Transplant 20: 532-538, 2005.

5. Brezniceanu ML, Liu F, Wei CC, et al: Attenuation of interstitial fibrosis and tubular apoptosis in $\mathrm{db} / \mathrm{db}$ transgenic mice overexpressing catalase in renal proximal tubular cells. Diabetes 57: 451-459, 2008

6. Nitti M, Furfaro AL, Patriarca S, et al: Human mesangial cells resist glycoxidative stress through an antioxidant response. Int $\mathbf{J}$ Mol Med 27: 213-219, 2011.

7. Khera T, Martin J, Riley S, Steadman R and Phillips AO: Glucose enhances mesangial cell apoptosis. Lab Invest 86: 566-577, 2006.

8. Ferri KF and Kroemer G: Organelle-specific initiation of cell death pathways. Nat Cell Biol 3: E255-E263, 2001.

9. Ron D: Translational control in the endoplasmic reticulum stress response. J Clin Invest 110: 1383-1388, 2002.

10. Sheikh-Ali M, Sultan S, Alamir AR, Haas MJ and Mooradian AD: Hyperglycemia-induced endoplasmic reticulum stress in endothelial cells. Nutrition 26: 1146-1150, 2010.

11. Liu G, Sun Y, Li Z, et al: Apoptosis induced by endoplasmic reticulum stress involved in diabetic kidney disease. Biochem Biophys Res Commun 370: 651-656, 2008. 
12. Kaneto H, Matsuoka TA, Nakatani Y, et al: Oxidative stress, ER stress and the JNK pathway in type 2 diabetes. J Mol Med (Berl) 83: 429-439, 2005.

13. Zhong Y, Wang JJ and Zhang SX: Intermittent but not constant high glucose induces ER stress and inflammation in human retinal pericytes. Adv Exp Med Biol 723: 285-292, 2012.

14. Lai E, Bikopoulos G, Wheeler MB, Rozakis-Adcock M and Volchuk A: Differential activation of ER stress and apoptosis in response to chronically elevated free fatty acids in pancreatic beta-cells. Am J Physiol Endocrinol Metab 294: E540-E550, 2008.

15. Pino SC, O'Sullivan-Murphy B, Lidstone EA, et al: CHOP mediates endoplasmic reticulum stress-induced apoptosis in Gimap5-deficient T cells. PLoS One 4: e5468, 2009.

16. Wang SH, Shih YL, Lee CC, et al: The role of endoplasmic reticulum in cadmium-induced mesangial cell apoptosis. Chem Biol Interact 181: 45-51, 2009.

17. Inagi R: Endoplasmic reticulum stress in the kidney as a novel mediator of kidney injury. Nephron Exp Nephrol 112: e1-e9, 2009.

18. Xu L, Han F, Mandal A, Rao GN and Zhang X: Diazoxide attenuates hypothermic preservation-induced renal injury via down-regulation of CHOP and caspase-12. Nephrol Dial Transplant 25: 3859-3867, 2010.

19. Marciniak SJ, Yun CY, Oyadomari S, et al: CHOP induces death by promoting protein synthesis and oxidation in the stressed endoplasmic reticulum. Genes Dev 18: 3066-3077, 2004.

20. Suzuki $\mathrm{H}$ and Matsuoka M: TDP-43 toxicity is mediated by the unfolded protein response-unrelated induction of C/EBP homologous protein expression. J Neurosci Res 90: 641-647, 2012.
21. Cox DJ, Strudwick N, Ali AA, Paton AW, Paton JC and Schroder M: Measuring signaling by the unfolded protein response. Methods Enzymol 491: 261-292, 2011.

22. Jauhiainen A, Thomsen C, Strömbom L, et al: Distinct cytoplasmic and nuclear functions of the stress induced protein DDIT3/CHOP/GADD153. PLoS One 7: e33208, 2012.

23. Myoishi M, Hao H, Minamino T, et al: Increased endoplasmic reticulum stress in atherosclerotic plaques associated with acute coronary syndrome. Circulation 116: 1226-1233, 2007.

24. Loughlin DT and Artlett CM: Precursor of advanced glycation end products mediates ER-stress-induced caspase-3 activation of human dermal fibroblasts through NAD $(\mathrm{P}) \mathrm{H}$ oxidase 4. PLoS One 5: e11093, 2010.

25. Porter AG and Janicke RU: Emerging roles of caspase-3 in apoptosis. Cell Death Differ 6: 99-104, 1999.

26. Gómez-Guerrero C, Hernández-Vargas P, López-Franco O, Ortiz-Muñoz G and Egido J: Mesangial cells and glomerular inflammation: from the pathogenesis to novel therapeutic approaches. Curr Drug Targets Inflamm Allergy 4: 341-351, 2005.

27. Rodriguez-Barbero A, L'Azou B, Cambar J and López-Novoa JM: Potential use of isolated glomeruli and cultured mesangial cells as in vitro models to assess nephrotoxicity. Cell Biol Toxicol 16: 145-153, 2000.

28. Chen P, Shi Q, Xu X, Wang Y, Chen W and Wang H: Quercetin suppresses NF- $\kappa \mathrm{B}$ and MCP-1 expression in a high glucose-induced human mesangial cell proliferation model. Int J Mol Med 30: 119-125, 2012. 